\title{
Operational Assessment of Multi-Tone Direct Sequence CDMA on Nakagami m-fading Channel
}

\author{
Yussuff Abayomi Isiaka O. ", Folorunsho Hamed B. \\ Lagos State University, Lagos, Nigeria \\ *e-mail: abayomi.yussuff@lasu.edu.ng
}

Received: $19^{\text {th }}$ September 2018

Accepted: $28^{\text {th }}$ December 2018

Published: $20^{\text {th }}$ March 2019

https://doi.org/10.47545/etrj.2019.4.1.048

\begin{abstract}
Multi-tone direct sequence code division multiple access (MT-DS-CDMA) is a promising technique to support multiple users with secured high speed wireless communications as it capable to improve the system performance by mitigating multiple access interference (MAI). In this research work, operational assessment of MT-DS-CDMA using binary phase shift modulation (BPSK) scheme over Nakagami m-fading channel (a generalized distribution which can model different fading environments to study the impact of fading channels on wireless communications) considering channel impairments has been analyzed. An analytical approach for basic communication signal transmission using BPSK modulator was used to find the expressions of signal to interference plus noise ratio (SINR) as well as BER with maximal ratio combining (MRC) in the presence of multiple-access interference (MAI) and noise. The assessment was carried out through simulation on Matlab. The system performance was evaluated considering SINR and BER by varying number of multiple users, fading parameter and number of sub-carriers. The assessment was carried out to find the effect of carrier signals to MAI, noise, and active users in MT DS CDMA communication scheme. The results presented that increase in number of active users required increase in number of sub-carriers, increase in MAI, increase in BER and increase in signal power. Again, an increase in signal power resulted in increased SINR. Furthermore, an increase in signal power led to decreased BER. Hence, it was concluded that Signal power and SINR are key component for consideration to optimize performance of MT-DS-CDMA.
\end{abstract}

Keywords: BER, BPSK, MAI, MT-DS-CDMA, NAKAGAMI FADING, SINR.

\section{INTRODUCTION}

Code Division Multiple Access (CDMA) has become a popular technology for cellular communications. The idea in CDMA system is to transmit signals simultaneously through a linear band-limited channel without interchannel or inter-symbol interference [1]. CDMA system utilizes the spread-spectrum technique, where the message signal can occupy both time and frequency domains simultaneously; thus the achieving significant increased system capacity [2,3]. Spread-spectrum is a transmission technique in which the transmitted signal occupies a bandwidth more than the minimum necessary to transmit the information. Band spreading is accomplished by generating an independent code. A reception that is synchronized to the code is used to despread and recover the data at the receiver end [3,4]. Coding is used to transform the information signal into noise-like form [5]. Spread spectrum communication scheme has many features that make it suitable for secure, multiple accesses and several other properties required communication systems. There are two types of Spread spectrum systems. These are: Frequency Hopping (FH) and Direct Sequence (DS) system. CDMA is a type of DS spread spectrum system (DS-CDMA). The general principle behind DS-CDMA is that the information signal with bandwidth $B_{S}$ is spread over a bandwidth $B$, where $B$ is much greater than $B_{S}[6,7]$. Resulting from researches in CDMA is the Orthogonal Frequency Division Multiplexing (OFDM). OFDM is a multicarrier modulation technique, that spreads the information to be transmitted onto several orthogonal sinusoidal subcarriers, making the bits on each subcarrier are much longer, and thus, drastically reducing the effect of any channel dispersion (which could cause intra channel and inter-symbol interference) [8]. OFDM improves system capacity by making transmission more robust to frequency selective fading; hence viewing the system capacity increase as enhancing system spectral efficiency $[7,8]$. 


\subsection{Concept of multicarrier modulation scheme}

Aggressive demand for higher data communication rates has led to efforts towards multicarrier modulation. Multicarrier CDMA (MC-CDMA) is a modulation technique that combines OFDM and CDMA. In multicarrier modulation, the information signal is split into several components and then each component is sent over different carrier signals known as sub-carriers [9]. Two common forms of MC-CDMA are Multi-tone DSCDMA (MT-DS-CDMA) and Multicarrier DS-CDMA (MC-DS-CDMA). In MT DS CDMA system, each data symbol is serial to parallel converted and then spread before being transmitted over different subcarriers; the subcarrier frequencies are orthogonal to each other with minimum frequency separations between them before DS spreading is undertaken.

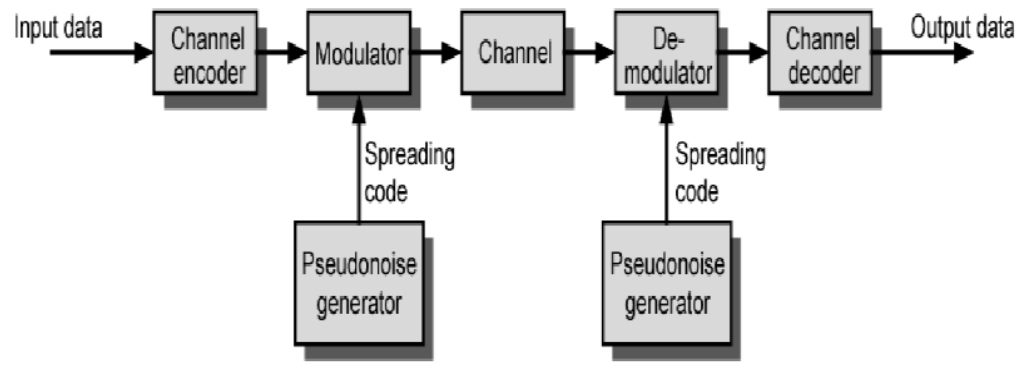

Figure 1: General Model of Spread Spectrum System [9].

\section{SYSTEM MODEL}

\subsection{Transmitter Model}

The transmitter model of MT-DS-CDMA with binary phase shift modulation (BPSK) modulator is described in Figure 2. The transmitter block shows MT-DS-CDMA with code generator, modulator, and a summer. The input data stream is converted into parallel data streams with bit duration, $T_{b}$. Parallel data symbols of each data stream of $i^{\text {th }}$ user (assuming $i^{\text {th }}$ user is transmitting) are coded by the code generator in pseudo-random noise (PN) sequence having chip duration, $T_{c}$ and then spread in the time domain. The spread data is then modulated by BPSK modulator with $N_{S C}$ subcarriers. Then a summer merges the modulated signals and a MT-DS-CDMA antenna transmits the signal through a multipath Nakagami-m fading wireless channel $[10,11]$.

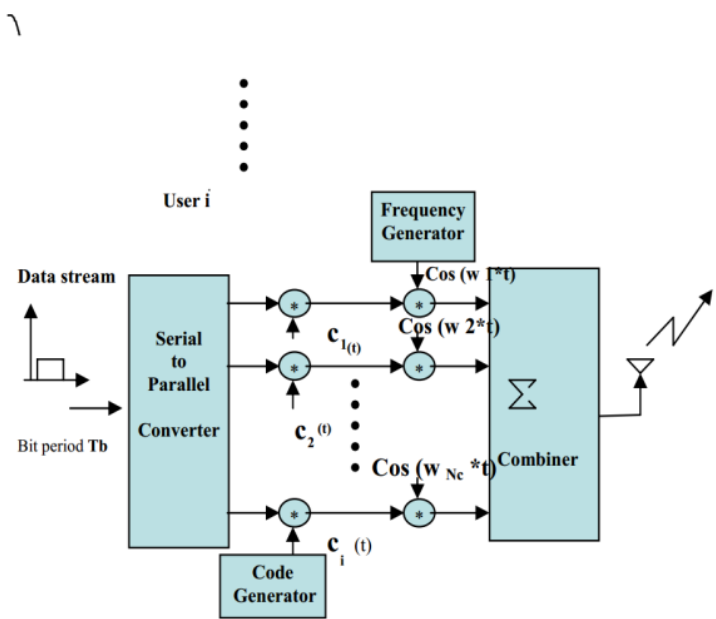

Figure 2: MT-DS-CDMA Transmitter Model [11]. 
The MT-DS-CDMA scheme uses longer spreading codes in proportion to the number of sub-carriers $\left(N_{S C}\right)$, compared with a normal (single-carrier) DS-CDMA scheme. The expression for the transmitted signal is:

$\mathrm{ST}(\mathrm{t})=\sum_{K=1}^{N_{c}}\left\{\sqrt{2 p} b_{n, k}^{j}\left[\sum_{k=1}^{N_{c}} C_{x, k}^{j}\right]\right\} \cos \left(\omega_{c} t+\mathrm{k} \Delta \omega+\varphi_{k}\right)$

Where, $b_{n, k}^{i}=\mathrm{n}^{\text {th }}$ bit of the $i^{\text {th }}$ user which is being modulated by the $k^{\text {th }}$ channel

$C_{x, k}^{i}=x^{t h}$ chip of the $k^{t h}$ section of the $i^{t h}$ users code.

Where ST(t) is the transmitted Signal, $P$ is the chip power of the user, $N_{S C}$ is the number of subcarrier channels and $N$ is the number of chips of the code for each subcarrier channel.

\subsection{Receiver model}

The receiver model of a MT-DS-CDMA with BPSK demodulator is described in Figure 3. The receiver block consists of parallel to serial converter for realizing the reverse operation of serial to parallel conversion at the transmitter, code degenerator, demodulator, low-pass filter (LPF), and receiver antenna. At reception, the receiver collects all the signals transmitted by $K$ number of users while the demodulator removes the carrier signal (demodulates) from the received signal with $N_{S C}$ sub-carriers. $[11,13]$

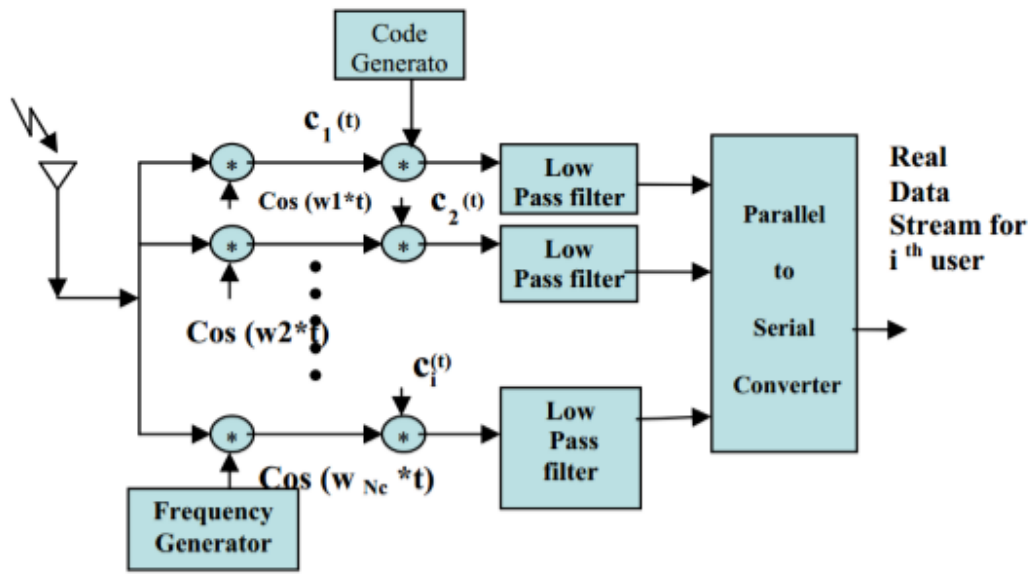

Figure 3: MT-DS- CDMA Receiver Model [11].

The received signal is a summation of data of $i^{\text {th }}$ number of users with some noise. The received signal equation is expressed as: ${ }^{13,14}$

$$
\mathrm{r}(\mathrm{t})=\sum_{m=1}^{j} \propto\left[\sqrt{2 p} \sum_{k=1}^{N_{c}} b_{n, k}^{m}\left[\sum_{x=1}^{N} C_{x, k}^{j}\right] \cos \left(\omega_{c} t+\mathrm{k} \Delta \omega_{c} \mathrm{t}+\varphi_{k}\right)+\eta(t)\right]
$$

Here, $\eta(t)$ is the additive white Gaussian noise (AWGN), whose spectral density is around $N_{0} / 2$. This signal, while propagating through the medium suffers frequency selective multi-path Nakagami-m fading. Hence, when considering that $\propto$ is the instantaneous amplitude distortion and, $\theta$ be the instantaneous phase distortion due to fading, the expression at the receiver end will be [14].

$$
\mathrm{r}(\mathrm{t})=\sum_{m=1}^{j} \propto\left[\sqrt{2 p} \sum_{k=1}^{N_{c}} b_{n, k}^{m}\left[\sum_{x=1}^{N} C_{x, k}^{j}\right] \cos \left(\omega_{c} t+\mathrm{k} \Delta \omega_{c} \mathrm{t}+\theta+\varphi_{k}+\delta \omega_{c} t\right)+\eta(t)\right]
$$

\section{THEORETICAL ANALYSIS}

\section{Demodulated signal}

$\mathrm{y}(\mathrm{t})=\sum_{m=1}^{i} \propto\left[\sqrt{2 p} \sum_{k=1}^{N_{s c}} b_{n, k}^{m}\left[\begin{array}{lll}\sum_{x=1}^{N} C_{x, k}^{i} & x & C_{x, k}^{i}\end{array}\right] \cos \left(\omega_{c} t+\mathrm{k} \Delta \omega_{c}+\theta+\varphi_{k}+\delta \omega_{c} t\right)+\cos \left(\omega_{c} \mathrm{t}+\right.\right.$ $\left.\left.\mathrm{k} \Delta \omega_{c} \mathrm{t}\right)\right]+\sum_{k=1}^{N_{s c}} \eta(t) x \cos \left(\omega_{c} \mathrm{t}+\mathrm{k} \Delta \omega_{c} \mathrm{t}\right)$ 


\section{Decoded signal}

$\mathrm{y} \quad(\mathrm{t})=\sum_{m=1}^{i}\left[\alpha \frac{\sqrt{2 p}}{2}\right] \sum_{k=1}^{N_{c}} b_{n, k}^{m}\left[\sum_{x=1}^{N} C_{x, k}^{i}\right] \cos \left(2 \omega_{c} t+2 \mathrm{k} \Delta \omega_{c} \mathrm{t}+\theta+\varphi_{k}+\delta \omega_{c} t\right)+\cos \left(\delta \omega_{c} t+\varphi_{k}+\right.$ $\theta)+\sum_{k=1}^{N_{s c}} \eta(t) \sum_{x=1}^{N} C_{x, k} \cos \left(\omega_{c} \mathrm{t}+\mathrm{k} \Delta \omega_{c} \mathrm{t}\right)$

\section{Passing through LPF.}

y $\quad(\mathrm{t}) \quad=\frac{N \propto \sqrt{2 p}}{2} \sum_{k=1}^{N_{s c}} b_{n, k}^{i} \cos \left(\delta \omega_{c} t+\varphi_{k}+\theta\right)+\sum_{m=1}^{i-1}\left[\frac{\alpha \sqrt{2 p}}{2} \sum_{k=1}^{N_{s c}}\left[b_{n, k}^{m} \mu N \cos \left(\delta \omega_{c} t+\quad \varphi_{k}+\right.\right.\right.$ $\theta)]]+\sum_{k=1}^{N_{s c}} \eta(t) \sum_{x=1}^{N} C_{x, k}^{i} \cos \left(\omega_{c} \mathrm{t}+\mathrm{k} \Delta \omega_{c} \mathrm{t}\right)$

$$
\begin{aligned}
& \text { Integrating over } 0 \text { to } \mathbf{T b} \\
& \mathrm{y}(\mathrm{t})=\frac{N \propto \sqrt{2 p}}{2 T_{b}} \int_{0}^{T_{b}}\left[\sum_{k=1}^{N_{s c}} b_{n, k}^{i} \cos \left(\delta \omega_{c} t+\varphi_{k}+\theta\right)\right] d t+\quad \sum_{m=1}^{i-1}\left[\frac { \alpha \sqrt { 2 p } } { 2 T _ { b } } \int _ { 0 } ^ { T _ { b } } \sum _ { k = 1 } ^ { N _ { s c } } \left[b _ { n , k } ^ { m } \mu N \operatorname { c o s } \left(\delta \omega_{c} t+\right.\right.\right. \\
& \left.\left.\left.\varphi_{k}+\theta\right)\right]\right] d t+\frac{1}{T_{b}} \int_{0}^{T_{b}}\left[\sum_{k=1}^{N} \eta(t) \sum_{x=1}^{N} C_{x, k}^{i} \cos \left(\omega_{c} \mathrm{t}+\mathrm{k} \Delta \omega_{c} \mathrm{t}\right)\right] d t
\end{aligned}
$$

\section{Result of integration}

$\mathrm{Y}(\mathrm{t})$

$$
\left.=\frac{N \propto \sqrt{2 p}}{2} \sum_{k=1}^{N_{s c}} b_{n, k}^{m} \cos \left(\varphi_{k}+\theta\right)+\sum_{m=1}^{i-1} \frac{\alpha \mu N \sqrt{2 p}}{2 T_{b}} \sum_{k=1}^{N_{s c}} b_{n, k}^{m} \cos \left(\varphi_{k}+\theta\right)\right]+
$$

$$
\frac{1}{T_{b}} \int_{0}^{T_{b}}\left[\sum_{k=1}^{N_{s c}} C_{x, k}^{i} \cos \left(\omega_{c} \mathrm{t}+\mathrm{k} \Delta \omega_{c} \mathrm{t}\right)\right] d t
$$

Hence,

$$
\mathrm{Y}(\mathrm{t})=Y_{i}(t)+Y_{M A I}(t)+\eta(t)
$$

Where,

$Y_{i}(t)=$ Desired signal of $\mathrm{i}^{\text {th }}$ user

$Y_{M A I}(t)=$ Multiple Access Interference

$\eta(t)=$ Noise.

\section{Desired signal}

$$
\mathrm{Y}_{\mathrm{i}}(\mathrm{t})=\mathrm{N} \propto \sqrt{\frac{P}{2}} \sum_{k=1}^{N_{c}} b_{n, k}^{i} \cos \left(\emptyset_{k}+\theta\right)
$$

but since, $\quad \mathrm{b}_{\mathrm{n}, \mathrm{k}}^{\mathrm{j}}= \pm 1$ and $\sum_{k=1}^{N_{c}} b_{n, k}^{j}=N_{C}$

$\mathrm{Y}_{\mathrm{i}}(\mathrm{t})=\mathrm{NN}_{\mathrm{c}} \propto \sqrt{\frac{P}{2}} \sum_{k=1}^{N_{c}} \cos \left(\varnothing_{k}+\theta\right)$

Signal power (Ps) is given as;

$$
\begin{aligned}
\mathrm{P}_{\mathrm{S}} & =\frac{1}{2} \alpha^{2}\left(\mathrm{NN}_{\mathrm{c}} \propto \sqrt{\frac{P}{2}}\right)^{2} \\
& =\frac{1}{4} \alpha^{2} \mathrm{~N}^{2} \mathrm{~N}_{\mathrm{c}}^{2} \mathrm{P}
\end{aligned}
$$

\section{Multiple Access Interference (MAI)}

$$
\mathrm{Y}_{\mathrm{MAI}}(\mathrm{t})=\sum_{m=1}^{j-1}\left[\frac{\propto N \mu \sqrt{2 P}}{2} \sum_{k=1}^{N_{c}} b_{n, k}^{m} \cos \left(\emptyset_{k}+\theta\right)\right]
$$

Interference power is given as; 


$$
\begin{aligned}
\mathrm{P}_{\mathrm{MAI}}(\mathrm{t}) & =\sum_{m=0}^{j-1}\left[\frac{1}{2} \alpha^{2} \mu^{2} N^{2} N_{c}{ }^{2} \frac{P}{2}\right] \\
& =\sum_{m=0}^{j-1}\left[\frac{1}{4} \alpha^{2} \mu^{2} N^{2} N_{s c}{ }^{2} P^{2}\right] \\
& =\left[\frac{1}{4} \alpha^{2} \mu^{2} N^{2} N_{s c}{ }^{2} P(j-1)\right]
\end{aligned}
$$

Noise

$\eta(t)=\frac{1}{T_{b}} \int_{0}^{T_{b}} \omega(t)\left[\sum_{k=1}^{N_{c}} C_{x, k}^{j} \cos \left(\omega_{c} \mathrm{t}+\mathrm{k} \Delta \omega_{c} \mathrm{t}\right)\right] d t$

Thus, evaluated Noise power is;

$\sigma_{n}^{2}=\frac{N_{0}}{4 T_{b}}$, where $\mathrm{N}_{0}=\mathrm{kTR}_{\mathrm{b}}$

$\mathrm{K}=$ Boltzmann constant, $\mathrm{T}=$ Temperature, $\mathrm{Rb}=$ Bits Rate

\section{SINR}

Signal to Noise and Interference Ratio (SINR) can be expressed as:

$$
\begin{aligned}
\text { SINR } & =\frac{P_{S}}{P_{M A I}+\sigma_{n}^{2}} \\
& ==\frac{\frac{1}{4} \alpha^{2} N^{2} N_{C}^{2} \mathrm{P}}{\frac{1}{4} \alpha^{2} \mu^{2} N^{2} N_{C}^{2} P(j-1)+\frac{N_{0}}{4 T_{b}}} \\
& =\frac{\alpha^{2} N^{2} N_{C}^{2} P T_{b}}{\alpha^{2} \mu^{2} N^{2} N_{C}^{2} P(j-1)+N_{0}} \\
& =\frac{\alpha^{2} N^{2} N_{C}^{2}}{\alpha^{2} \mu^{2} N^{2} N_{C}{ }^{2}(j-1)+\left(\frac{E_{b}}{N_{0}}\right)^{-1}}
\end{aligned}
$$

\section{Bit Error Rate (BER)}

SINR is a function of $\alpha$. So for an instantaneous value of $\alpha$, the instantaneous Bit error rate (BER) is:

$$
=\frac{1}{2} \operatorname{erf} C\left[\sqrt{\frac{\operatorname{SINR}}{2}}\right]
$$

Thus,

$$
\mathrm{BER}=0.5 x \operatorname{erf} C\left[\frac{\alpha^{2} N^{2} N c^{2}(1 / \sqrt{2})}{\alpha^{2} \mu^{2} N^{2} N c^{2}(j-1)+\left(E b / N_{0}\right)^{-1}}\right]
$$

Now the average bit error rate of the system is:

$\mathrm{BER}=\int_{0}^{\infty} \mathrm{P}_{\mathrm{b}}(\propto) \times \mathrm{f}(\propto) d \propto$

Thus, $\quad \operatorname{BER}(\alpha)=\sum_{i=1}^{N} \mathrm{P}_{\mathrm{b}}\left(\propto_{i}\right) \times f\left(\propto_{i}\right)$

\section{RESULTS AND DISCUSSION}

Following the theoretical analysis above. Basic equations for MAI, SINR, NOISE, and BER were simulated on Matlab and results were obtained.

Figure 4, shows the plot of the signal to interference and noise ratio (SINR) against the number of users $(N)$. The Figure is obtained from Equation 15. It is observed that increase in N produces a decrease in the SINR. The SINR depends on signal power $\left(P_{S}\right)$. Here $P_{S}$ is $1 \mathrm{mw}$ and the curve is for the number of sub-carrier $\left(N_{S C}\right)=4$, and the instantaneous BER $(\alpha)$, is 1 . 


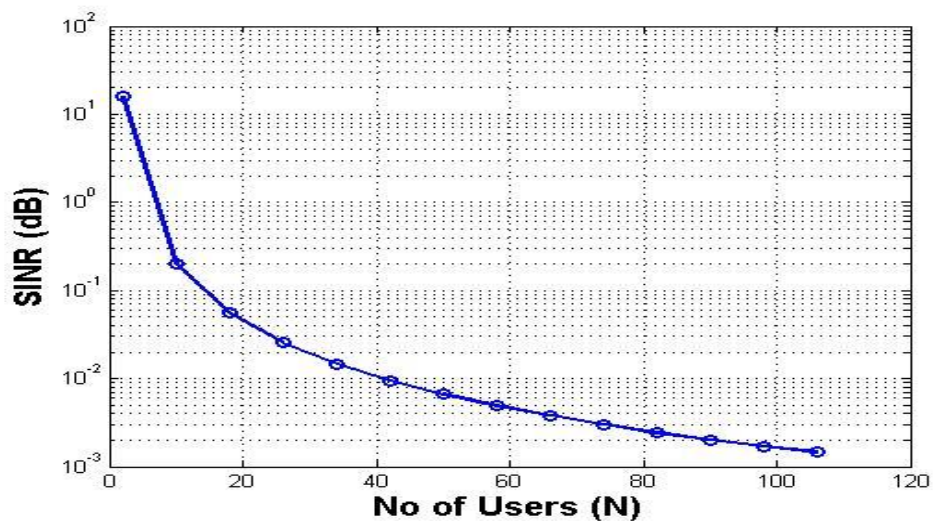

Figure 4: Plot of SINR versus $N$

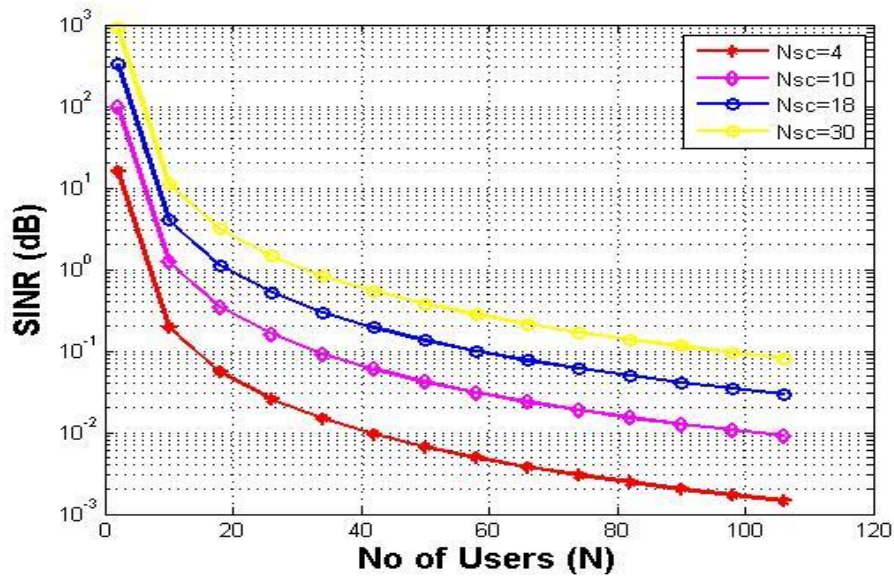

Figure 5: Plot of $S I N R$ versus $N$ (with different $N_{S C}$ values)

Figure 5, is the modified version of Figure 4. The Figure is also obtained from Equation 15. Here, the number of subscribers were varied as $N_{S C}=4,10,18,30$. The plot also exhibits the same trait observed in Figure 4 (that is, as $N$ increases, the $S I N R$ decreases).

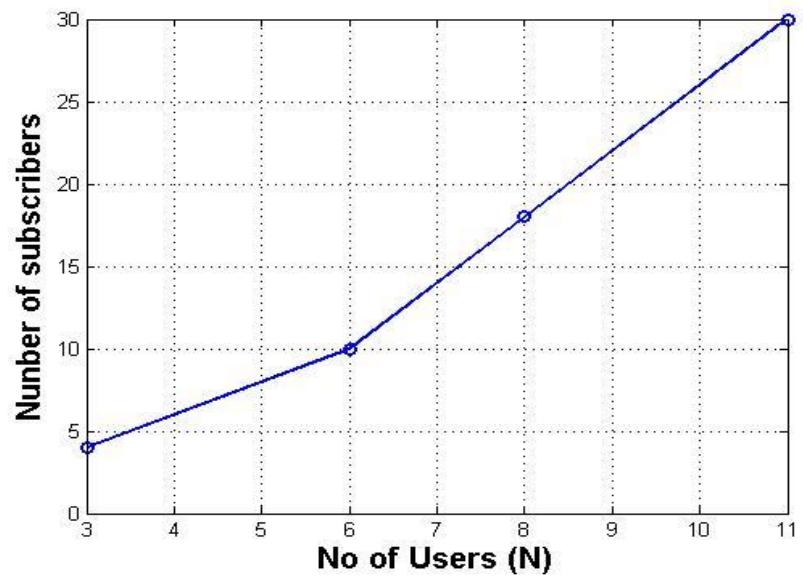

Figure 6: Plot of $N_{S C}$ versus $N$

Again, Figure 6 illustrates the plot of the number of sub-carrier $\left(N_{S C}\right)$ against the number of users $(N)$. The figure was obtained from Equation 10 by varying $N_{S C}$ and $\mathrm{N}$. It is observed that increase in the numbers of user 
$(N)$ requires increase in number of sub-carriers $\left(N_{S C}\right)$; the major consequence is that an increase in the number of users that can transmit simultaneously requires an increase the number of sub-carriers. This could be used as a measure to determine the capacity of MT-DS-CDMA system.

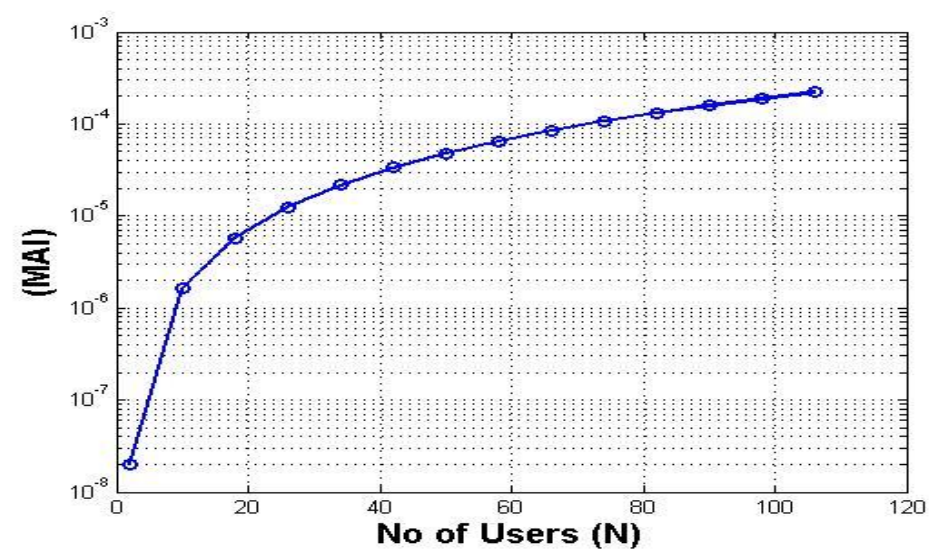

Figure 7: Plot of MAI versus $N$

Furthermore, Figure 7 displays the plot of Multiple Access Interference (MAI) against number of users $(N)$. The Figure was realized from Equation 12. It is observed that as number of users increase there is a corresponding increase in MAI, meaning that, an increase in the number of users results in increase in interference of subcarriers $\left(N_{S C}\right)$. Hence, it is concluded that MAI is subject to cross-correlation properties of spreading code, number of users $(N)$, instantaneous BER $(\alpha)$, and number of sub-carriers $\left(N_{S C}\right)$.

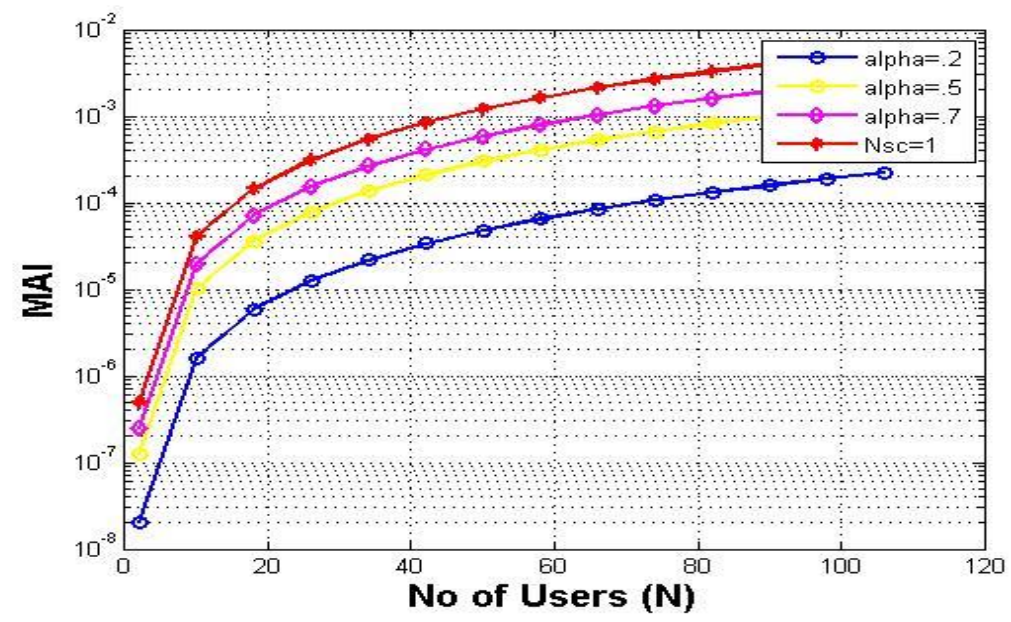

Figure 8: Plot of MAI versus $N$ (with different value of $\alpha$ )

Still, Figure 8 indicates the plot of Multiple Access Interference (MAI) against Number of users $(N)$ (with different values of instantaneous BER, $\alpha$ ). The Figure is developed using Equation 12. The curves was plotted for different values of instantaneous BER, $a=0.2,0.5,0.7,1$. The signal power $\left(P_{S}\right)=1 \mathrm{mw}$ and the $N=14$. It is observed that as alpha increase MAI increase. This reinforces the assertion that MAI is subject to increase in $a$, cross-correlation properties of spreading code, $N$, and $N_{S C}$.

Figure 9, shows the plot of Amplitude distortion due to fading (a) versus $N$. The Figure is obtained from Equation 10. Again, It is observed that as $N$ increase $\alpha$ decreases. Here, multiple access interference is considered as $10^{5}$. The implication is that amplitude distortion due to fading is independent of number of users transmitting simultaneously. Hence, it is concluded that $\alpha$ is independent of number of users transmitting in a wireless network 


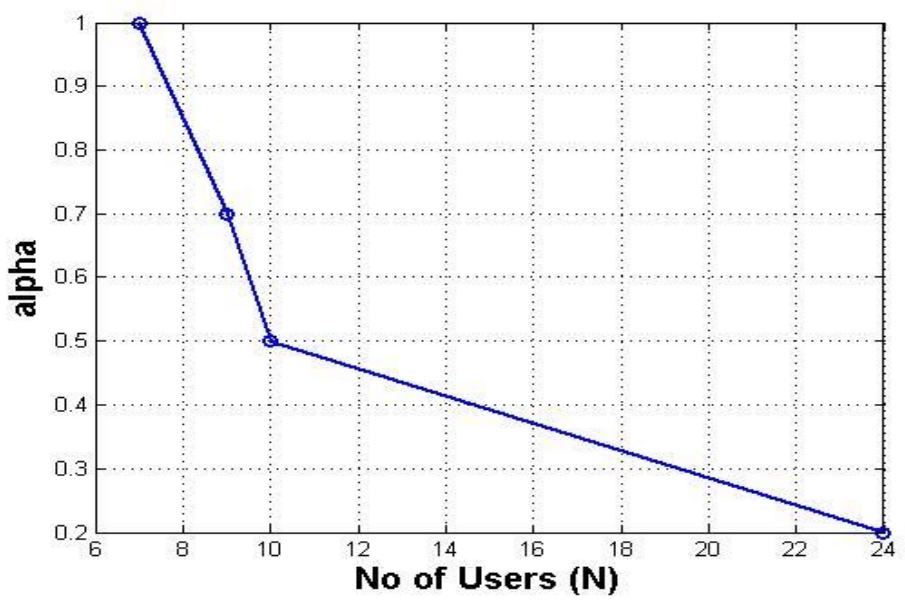

Figure 9: Plot of $a$ against $N$

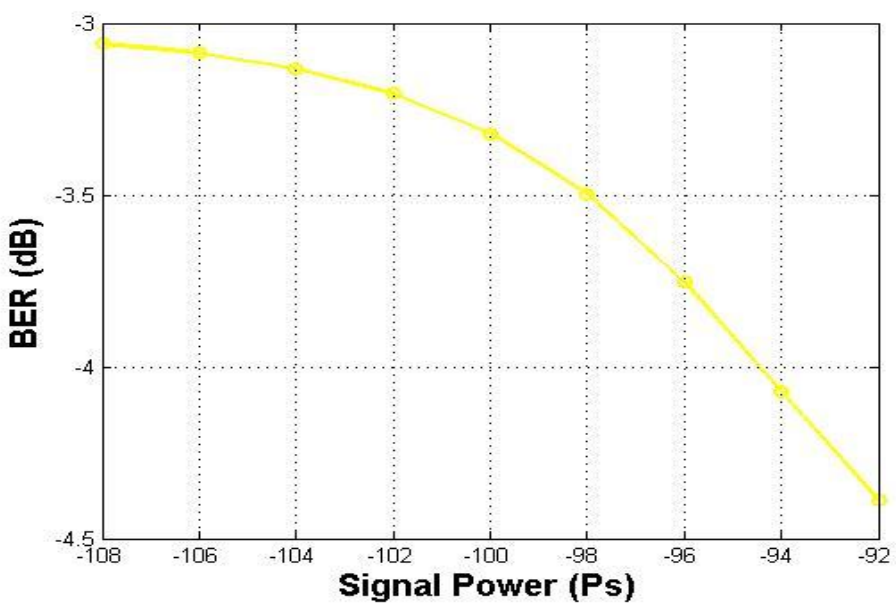

Figure 10: Plot of BER versus $P_{S}$

Moreover, Figure 10 shows the plot of (BER in dB) against signal power $\left(P_{S}\right)$. This Figure is obtained from Equation 16. Here, $a=0.2, N_{S C}=4$, and $N=10$. It is observed that as $P_{S}$ increases, BER decreases, indicating that BER is dependent on the power spectrum of a MT-DS-CDMA communication system. Likewise, the effect was also observed when varying values of fading as $a=0.2,0.5,0.7,0.9,1.0$. Because increase in $P_{S}$ produces a decrease in BER, it can be concluded that amplitude distortion signals in MT-DS-CDMA system has marginal or no effect. Also, BER is dependent on signal power, $P_{S}$.

Shown in Figure 11, is the plot of unconditional BER against $N$. The Figure is realized from Equation 18, where $N=128$ and each user is divided into 1000; hence the length of each gap here is $\delta a$. Once more, it was observed that as number of users increase the BER also increase and BER become fixed at a particular point of increase in $N$. This implies that BER is dependent on the number of users.

In Figure 12, BER was plotted against SINR. The Figure was obtained from Equation 16. It is observed that as SINR increase BER decrease. The implication is that BER is dependent on SINR. 


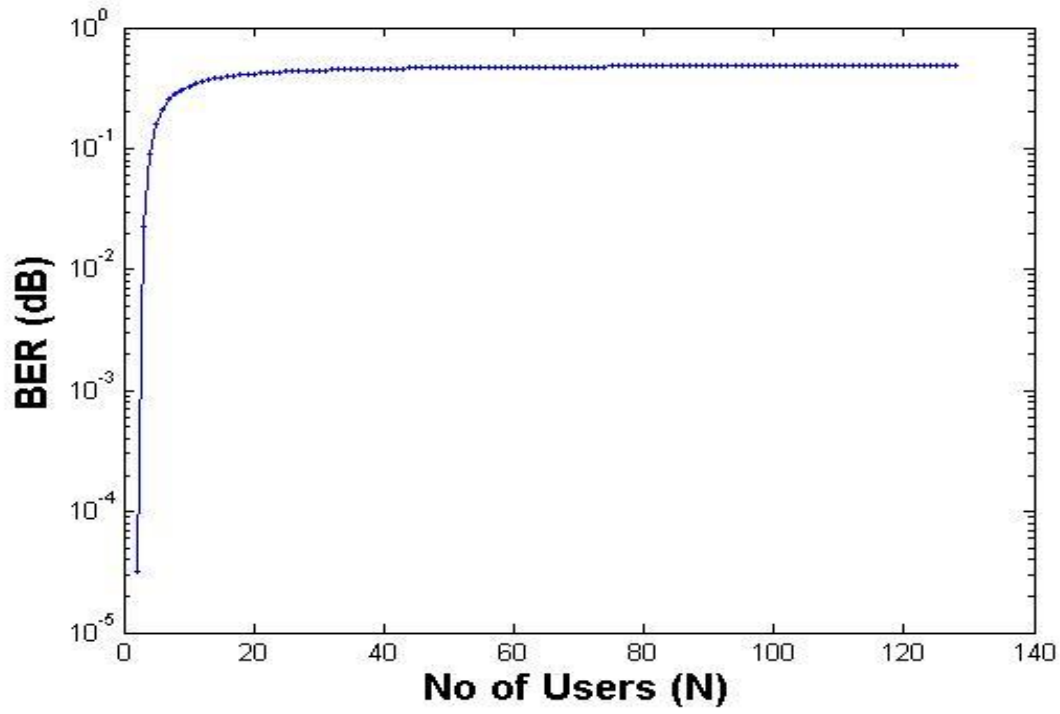

Figure 11: Plot of BER versus $N$

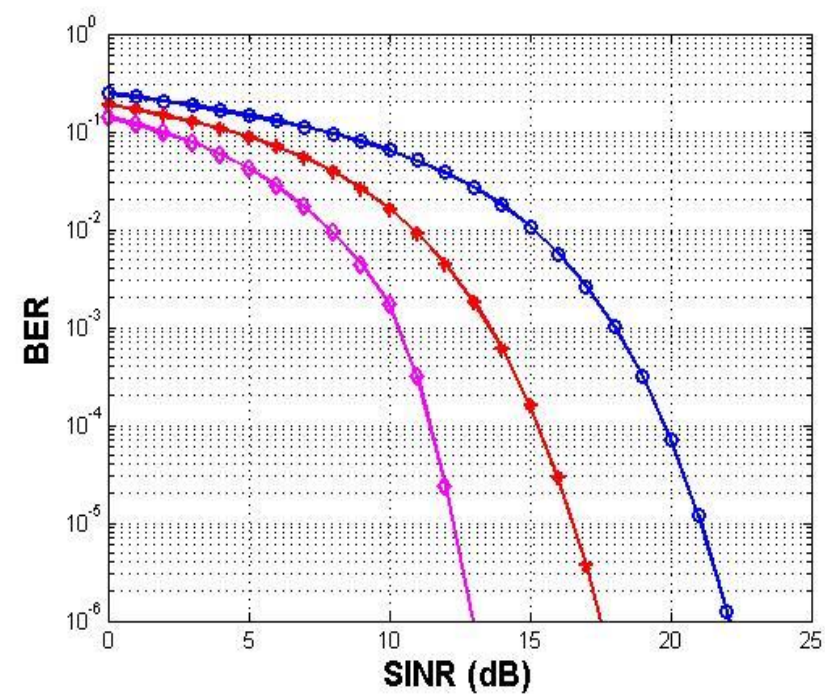

Figure 12: Plot BER versus SINR

\section{CONCLUSION}

This work presented operation assessment of MT-DS-CDMA system transmitting over Nakagami m-fading channel by applying BPSK modulation technique. The system behaviour's were scrutinized using BER, MAI, and SINR by varying number of users, number of subcarriers, and fading coefficient. It was observed that the performance of the MT-DS-CDMA system can be severely affected (increased BER) by increase in number of users without a corresponding increase in number of sub-carriers. Furthermore, that system power spectrum impacts on BER, which is a function of SINR. Again, an increase in SINR results in increase in BER but a low BER is expected for good quality of service. Hence, there is need for conscious balance between BER and SINR. Also, the relationship between SINR and signal power revealed that an increase in signal power will result in increase in SINR. An increase in the number of users indicates increase in MAI. Finally, it was observed that increase in number of users requires increase of signal power. 


\section{REFERENCES}

[1] S. Kaiser, "Potential of code division multiplexing (CDM) and spatial diversity in future broadband OFDM systems," in Proc. IEEE Wireless Communications and Networking Conference (WCNC 2008), Las Vegas, USA, March/April 2008.

[2] S. Kaiser, "OFDM with code division multiplexing and transmit antenna diversity for mobile communications," in Proc. IEEE International Symposium on Personal, Indoor and Mobile Radio Communications (PIMRC 2000), London, UK, pp. 804-808, Sept. 2000.

[3] D. W. Matolak, F. A. Alder "Comparing MT, MC, and SC DS-SS System Parameters for Equal Bandwidth and Equal Data Rate" Version 2, January 2002.

[4] H. H. Chen, "On next generation CDMA technology for future wireless networking," in Proc. IEEE Global Telecommunications Conference Workshops, Nov. 2004, pp. 120-129.

[5] T.Ojanpera and R.Prasd,"An overview of air interface multiple access for MT2000/UMTS, IEEEcommun.Mag” vol.38, no.10, pp.82-95, Sep.1998.

[6] L Vandendrope," Multitone Direct Sequence CDMA system in an Indoor wireless Environment," Proc. of IEEE first symposium of communications and vehicular technology in the Benelux, Delft, The Netherlands, Oct.1993, pp.4.1-.1-4.1.8.

[7] DS-CDMA, MC-CDMA and MT-CDMA for Mobile Multi-Media Communications - Vehicular Technology Conference, 1996. Mobile Technology for the Human Race', IEEE 46th. [8] Shinsuke Hara,Ramjee Prasad, Overview Of Multicarrier CDMA -IEEE Communications Magazine

[9] Kohno, Ryuji, Reuven Meidan, and Laurence B. Milstein. "Spread Spectrum Access Methods for Wireless Communications", IEEE Communication Magazine, Vol. 33, Issue 1, January 1995, Page(s) 58-67.

[10] Jachimczyk, Witold. "Spread Spectrum Tutorial”, Available at http://webpages.charter.net/witek/

[11] 978-1-4244-6632-0/10/\$26.00 C2010 IEEE -Performance analysis of Multi- tone CDMA wireless communication system, most secure communication for $4 \mathrm{G}$.

[12] N.Yee,J-P,Linnartz and G.Fettweis,"Multicarrier CDMA in indoor Wireless Radio Networks,"Proc.of IEEE PIMRC'93,Yokohama,Japan,September 1993,pp.109-113.

[13] P. W. Fu, and K.C. Chen, "Multi-rate MC-DS-CDMA with multiuser detections for wireless multimedia communications" Proc. IEEE VTC 2002, pp. 1536-1540, 2002.

[14] S. Kaiser, "Effects of channel estimation errors on spatial pre-coding schemes with phase flipping," in Proc. IEEE Vehicular Technology Conference (VTC 2007 Spring), Dublin, Ireland, April 2007. 